\title{
Feasibility, Safety, and Periprocedural Complications Associated with Endovascular Treatment of Ruptured Intracranial Aneurysms according to the Depth of Anesthesia
}

\author{
(D). Song, (D) C.-Y. Lee, and (D)H.-W. Kim
}

\begin{abstract}
BACKGROUND AND PURPOSE: The aim of the present study was to report the feasibility, safety, and periprocedural complications associated with EVT of ruptured intracranial aneurysms according to the depth of anesthesia. In most centers, endovascular treatment of intracranial aneurysm is performed under general anesthesia.
\end{abstract}

MATERIALS AND METHODS: Between March 2011 and December 2016, a total of 183 consecutive patients with 183 aneurysms were treated endovascularly at the authors' center. The data about the depth of anesthesia (local anesthesia, conscious sedation, deep sedation, and general anesthesia), procedural details, and clinical and radiologic outcomes were reviewed.

RESULTS: A total of 183 consecutive patients with 183 aneurysms (mean age, $60.2 \pm 14.8$ years; 54 men and 129 women) were successfully treated. Of these, 70 (38.3\%) patients underwent endovascular treatment under local anesthesia, 33 (18.0\%) patients underwent endovascular treatment under conscious sedation, 78 (42.6\%) patients underwent endovascular treatment under deep sedation, and only 2 (1.1\%) patients underwent endovascular treatment under general anesthesia. For patients who presented with Hunt and Hess grades 1, 2, 3, 4, and $5,75 \%, 59.6 \%, 59.1 \%, 53.3 \%$, and $35.3 \%$ were treated under local anesthesia or conscious sedation, respectively. The procedure-related complication rates amounted to $8.7 \%$ (16/183, with 11 thromboembolic complications and 5 intraprocedural ruptures) overall, and $7.7 \%$ (14/183) of complications were symptomatic events. In the patients with good clinical grade (Hunt and Hess 1 or 2), the procedure-related complication rate was 4.1\% (4/97), and all complications were symptomatic events under local anesthesia or conscious sedation.

CONCLUSIONS: In the authors' experience, local anesthesia or conscious sedation seemed safe and feasible for the patients with good clinical grade $\mathrm{SAH}$.

ABBREVIATIONS: EVT = endovascular treatment; $\mathrm{HH}=$ Hunt and Hess scale

th $\mathrm{n}$ the past decade, endovascular treatment (EVT) has evolved to the mainstream treatment for the patients with ruptured intracranial aneurysms. In most centers, EVT of aneurysms is performed with the patient under general anesthesia to ensure control of hemodynamic and respiratory profiles, improved image quality in an immobile patient, and patient comfort. ${ }^{1-3}$ However, general anesthesia has potential risk of respiratory and hemodynamic instability during induction, interrupting the neurologic

Received April 13, 2018; accepted after revision June 6.

From the Department of Neurosurgery, College of Medicine, Konyang University Hospital, Konyang University Myunggok Medical Research Institute, Daejeon, Republic of Korea.

Please address correspondence to Cheol-Young Lee, MD, Department of Neurosurgery, College of Medicine, Konyang University Hospital, Konyang University Myunggok Medical Research Institute, 58, Gwanjeodong-ro, Seo-gu, Daejeon, Republic of Korea; e-mail: milelon@kyuh.ac.kr

三 Indicates article with supplemental on-line table.

http://dx.doi.org/10.3174/ajnr.A5753 examination during the procedure and causing respiratory complications due to mechanical ventilation. ${ }^{3,4}$

Sedation is a drug-induced depression of consciousness, a continuum culminating in general anesthesia. The American Society of Anesthesiology defines 3 levels of sedation and general anesthesia (Table 1). Minimal sedation is a depressed level of consciousness in which the patient retains the ability to respond normally to verbal commands. Conscious sedation is the level at which the patient can respond purposefully to verbal commands or tactile stimulation. Deep sedation allows the patient to respond to painful stimulation. Patients may require assistance in maintaining the airway and ventilation. General anesthesia is a loss of consciousness in which patients are not arousable and require assistance in maintaining the airway and positive pressure ventilation. Cardiovascular function may be impaired.

At the authors' center, we considered EVT with the patient under local anesthesia or conscious sedation first, according to the patient's 
Table 1: Continuum of depth of sedation-definition of general anesthesia and levels of sedation/analgesia

\begin{tabular}{|c|c|c|c|c|}
\hline & $\begin{array}{l}\text { Minimal Sedation/ } \\
\text { Anxiolysis }\end{array}$ & $\begin{array}{l}\text { Moderate Sedation/Analgesia } \\
\text { (Conscious Sedation) }\end{array}$ & Deep Sedation Analgesia & $\begin{array}{l}\text { General } \\
\text { Anesthesia }\end{array}$ \\
\hline Responsiveness & $\begin{array}{l}\text { Normal response to verbal } \\
\text { stimulation }\end{array}$ & $\begin{array}{l}\text { Purposeful }{ }^{a} \text { response to verbal } \\
\text { or tactile stimulation }\end{array}$ & $\begin{array}{l}\text { Purposeful } l^{a} \text { response after repeat } \\
\text { or painful stimulation }\end{array}$ & $\begin{array}{l}\text { Unarousable even with } \\
\text { painful stimulus }\end{array}$ \\
\hline Airway & Unaffected & No intervention required & Intervention may be required & $\begin{array}{l}\text { Intervention often } \\
\text { required }\end{array}$ \\
\hline Spontaneous ventilation & Unaffected & Adequate & May be inadequate & Frequently inadequate \\
\hline Cardiovascular function & Unaffected & Usually maintained & Usually maintained & May be impaired \\
\hline
\end{tabular}

${ }^{a}$ Reflex withdrawal from a painful stimulus is not considered a purposeful response.

clinical SAH grade. The purpose of this study was to examine the feasibility and safety of the use of local anesthesia or conscious sedation for EVT of ruptured intracranial aneurysms.

\section{MATERIALS AND METHODS Patient Population}

The present study was approved by our institutional review board. A total of 388 consecutive cases were treated with EVT in our institution from March 2011 to December 2016. Of these, 203 procedures were unruptured and 185 procedures were ruptured intracranial aneurysms. The patients with ruptured aneurysms who underwent EVT during the study period were included. Two patients who had no imaging follow-up were excluded. The remaining 183 patients were included in this study.

\section{Baseline Characteristics}

We retrospectively reviewed the patients' medical records, focusing on the following characteristics: sex, age, multiplicity of aneurysms, location, size of aneurysms, procedure details (eg, devices, coils, and medications used for anesthesia), procedure-related complications, procedure outcome with regard to technical success and underlying reasons for unsuccessful procedures, image findings after postoperative CT or MR imaging, procedure-related mortality and morbidity, and mRS score (at discharge and at 1 and 6 months).

\section{Anesthetic Technique and Patient Selection}

For the patients who did not require airway control because they were sufficiently alert and stable to undergo the procedure in the awake condition, local anesthesia or conscious sedation was administered. The patients who were in poor neurologic status (Glasgow Coma Scale Score $\leq 8$ at the time of the procedure) or unable to undergo the procedure in the awake state underwent deep sedation or general anesthesia. The information about the process of EVT was repeated and reinforced at the time of treatment by the nursing staff, technicians, and treating physicians. In our experience, this approach enhances the patient's cooperation throughout the various stages of the intervention.

Before each intervention, a Foley catheter and 2 intravenous lines were inserted in each patient in the intensive care unit. For a further reduction of head motion during the intervention, a custom-made, rigid, radiolucent headholder attached to the angiography table was used (Fig. 1). Electrocardiography, blood pressure cuffs, and pulse oximetry were applied throughout the procedure to monitor the patients. For real-time clinical assessment, frequent neurologic examinations (motor, sensory, and speech) were performed. In all procedures performed with the patient under local anesthesia, conscious sedation, or deep sedation, to

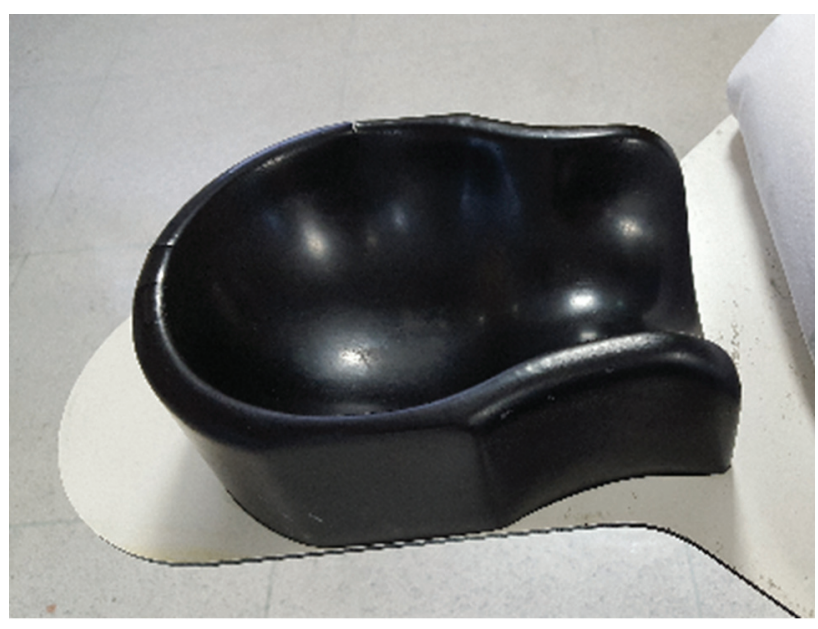

FIG 1. Rigid and radiolucent head holder.

ensure the possibility to convert to general anesthesia if needed (eg, due to the patient's intolerance, deterioration, or intraprocedural complications), an anesthesiologist was always available. After the intervention, all patients were returned to the intensive care unit for monitoring of periprocedural complications and vasospasm. Noncontrast CT was performed immediately after the procedure, and diffusion MR imaging was performed on the day after procedure to evaluate ischemic or hemorrhagic complications.

\section{Definitions and Outcome Variables}

Levels of sedation and anesthesia were defined according to the American Society of Anesthesiologists (Table 1). Local anesthesia is infiltration of local anesthetics into the groin without conscious sedation. Conscious sedation is a drug-induced depression of consciousness during which patients respond purposefully to verbal commands, either alone or accompanied by light tactile stimulation. Drugs were administered by a neurosurgeon. They consisted of an intravenous injection of $1 \mathrm{mg}$ of midazolam and/or 50 $\mathrm{mg}$ of fentanyl. To ensure that the patients could still respond to commands while resting comfortably, we administered an additional dose of midazolam when necessary (Ramsay sedation scale score of 2 or 36). Deep sedation is a drug-induced depression of consciousness during which patients cannot be easily aroused but are able to respond to repeat or painful stimulation. It was induced by intravenous injection of propofol $(40 \mathrm{mg})$. General anesthesia is a drug-induced loss of consciousness during which patients are not arousable, even by painful stimulation. Intravenous general anesthetic drugs were administered (fentanyl or midazolam) and, to achieve a concomitant neuromuscular blockade, pancuronium was used under the direction of the operating 
neurosurgeon. Infrequently, an anesthesiologist administered inhalational anesthetic agents.

Any undesirable events and/or abnormal angiographic findings related to the procedures were recorded, regardless of the symptom development, as procedure-related complications. In such cases, the neurosurgeon thoroughly reviewed procedural records and charts. We defined hemorrhagic complications as intraoperative rupture and thromboembolic complications as any thrombus formation during the procedure or abnormal findings on diffusion MR imaging regardless of the symptom development.

\section{Statistical Analysis}

The data were analyzed using the $\mathrm{R}$ statistical and computing software (http://www.r-project.org/). Continuous data were expressed as the mean \pm SD. The $\chi^{2}$ test or Fisher exact test was used to compare categoric values. Logistic regression models were used to evaluate independent associations between significant variables and treatment type. The odds ratio and 95\% confidence interval were calculated. A $P$ value $<.05$ was considered significant.

\section{RESULTS}

\section{Characteristics of Patients and Aneurysms}

During the study period, 183 consecutive patients with 183 aneurysms (mean age, $60.2 \pm 14.8$ years; range, 30-92 years; 54 men and 129 women) were successfully treated. Of these, 31 (16.9\%) patients had multiple aneurysms. Eighty (43.7\%) aneurysms were in the anterior cerebral artery, and 68 (39.9\%) aneurysms were $<5 \mathrm{~mm}$ in maximal diameter. Ninety-seven (53\%) patients were Hunt and Hess grade 1 or 2, 52 (28.4\%) patients were 3 or 4, and $34(18.6 \%)$ patients were grade 5 . One hundred three (56.3\%) patients underwent EVT under local anesthesia or conscious sedation, and only $2(1.1 \%)$ patients underwent EVT under general anesthesia (Table 2). Figure 2 demonstrates the distribution of patients according to clinical grade and depth of anesthesia.

\section{Aborted Procedures, Periprocedural Complications, and Outcomes}

All 183 procedures were successfully completed. Procedure-related complications occurred in $16(8.7 \%)$ of 183 procedures, including 11 thromboembolic (6\%) and 5 hemorrhagic $(2.7 \%)$ complications. Fourteen (7.7\%) complications were symptomatic events. Among 11 thromboembolic complications, 3 procedures were performed with the patient under local anesthesia, 2 were performed with the patient under conscious sedation, and 6 were performed with the patient under deep sedation. Two thromboembolic complications resolved with intra-arterial abciximab (ReoPro) injection without clinical symptoms and imaging abnormalities. Intraoperative perforation occurred in 5 procedures: 3 with the patient under conscious sedation, and 2 , under deep sedation. One of the patients developed an increased SAH requiring craniectomy and surgical evacuation, 2 patients died, and 2 patients had clinical deterioration (only 1 patient was asymptomatic). At the last follow-up (average, 12.4 months), 21 of the 183 patients had died; of these 21 , two had
Table 2: Baseline demographic data

\begin{tabular}{lc}
\multicolumn{1}{c}{ Variable } & No. \\
\hline No. of aneurysms & 183 \\
No. of patients & 183 \\
Age (mean) (SD) (yr) & $60.2(14.8)$ \\
Younger than 40 & $13(7.1 \%)$ \\
$40-50$ & $44(24.0 \%)$ \\
$50-60$ & $43(23.5 \%)$ \\
$60-70$ & $26(14.2 \%)$ \\
$70-80$ & $40(21.9 \%)$ \\
Older than 80 & $17(9.3 \%)$ \\
Sex & \\
Female & $129(70.5 \%)$ \\
Male & $54(29.5 \%)$ \\
Multiplicity & $31(16.9 \%)$ \\
Location & \\
ACA & $80(43.7 \%)$ \\
ICA & $56(30.6 \%)$ \\
MCA & $31(16.9 \%)$ \\
VB & $15(8.2 \%)$ \\
Aneurysm size (mm) & \\
55 & $68(39.9 \%)$ \\
$5-10$ & $92(47.5 \%)$ \\
$10-15$ & $21(11.5 \%)$ \\
>15 & $2(1.1 \%)$ \\
Modalities of EVT & \\
Simple catheter & $114(62.3 \%)$ \\
Double catheter & $47(25.7 \%)$ \\
Stent-assisted & $22(12.0 \%)$ \\
Anesthesia grade & \\
Local anesthesia & $70(38.3 \%)$ \\
Conscious sedation & $33(18.0 \%)$ \\
Deep sedation & $78(42.6 \%)$ \\
General anesthesia & $2(1.1 \%)$ \\
Hunt and Hess scale grade & \\
1 & $40(21.9 \%)$ \\
2 & $57(31.1 \%)$ \\
3 & $22(12.0 \%)$ \\
4 & $30(16.4 \%)$ \\
5 & $34(18.6 \%)$ \\
\hline
\end{tabular}

Note:-ACA indicates anterior cerebral artery; VB, vertebrobasilar artery.

an intraprocedural rupture and 1 had a thromboembolic event (mortality rate of $11.5 \%$ ). The mortality rate in the local anesthesia or conscious sedation group amounted to $1 \%$ ( 1 of 103 patients) (On-line Table).

\section{Clinical Grade and the Depth of Anesthesia}

For the patients who presented with Hunt and Hess grades 1, 2, 3, 4 , and $5,75 \%, 77.2 \%, 59.1 \%, 53.3 \%$, and $35.3 \%$ were treated under local anesthesia or conscious sedation, respectively (Fig 1). In patients with good clinical grade (Hunt and Hess grade 1 or 2), $76.3 \%$ (74/97) were treated under local anesthesia or conscious sedation. In patients with Hunt and Hess $(\mathrm{HH})$ grades 3,4 , or 5 , $33.7 \%(29 / 86)$ were treated under local anesthesia or conscious sedation. Among the 183 procedures, 16 procedures had procedure-related complications. Six patients $(6.2 \%)$ were HH 1 or 2 , and 4 complications occurred with the patient under local anesthesia or conscious sedation. Ten patients $(10 / 86,11.6 \%)$ were $\mathrm{HH} 3$, 4, or 5, and 4 complications occurred under local anesthesia or conscious sedation (Fig 3). There was no statistically significant difference between the rates of overall adverse events and clinical or angiographic factors (Table 3 ). 


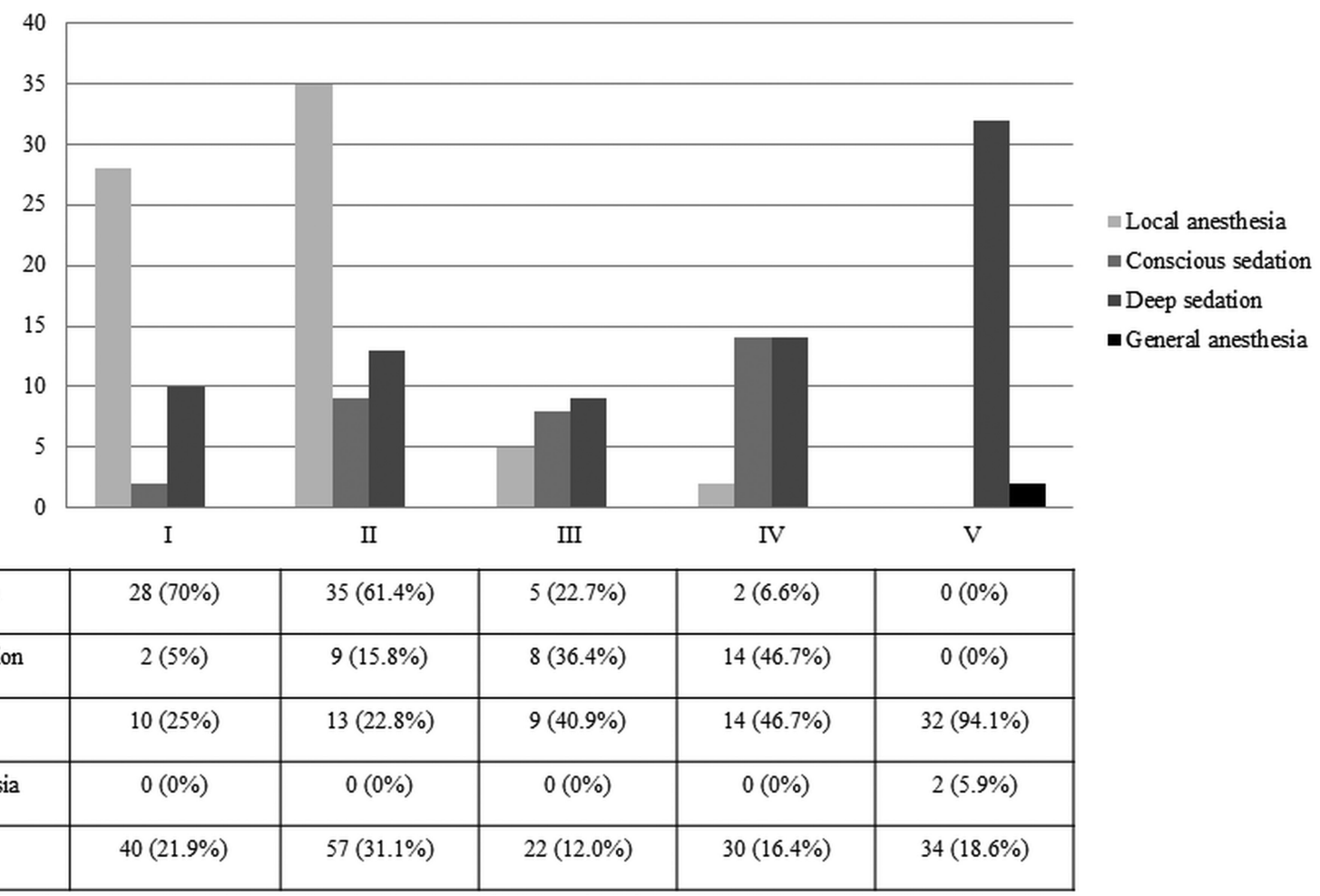

FIG 2. Distribution of patients according to clinical grade and depth of anesthesia.

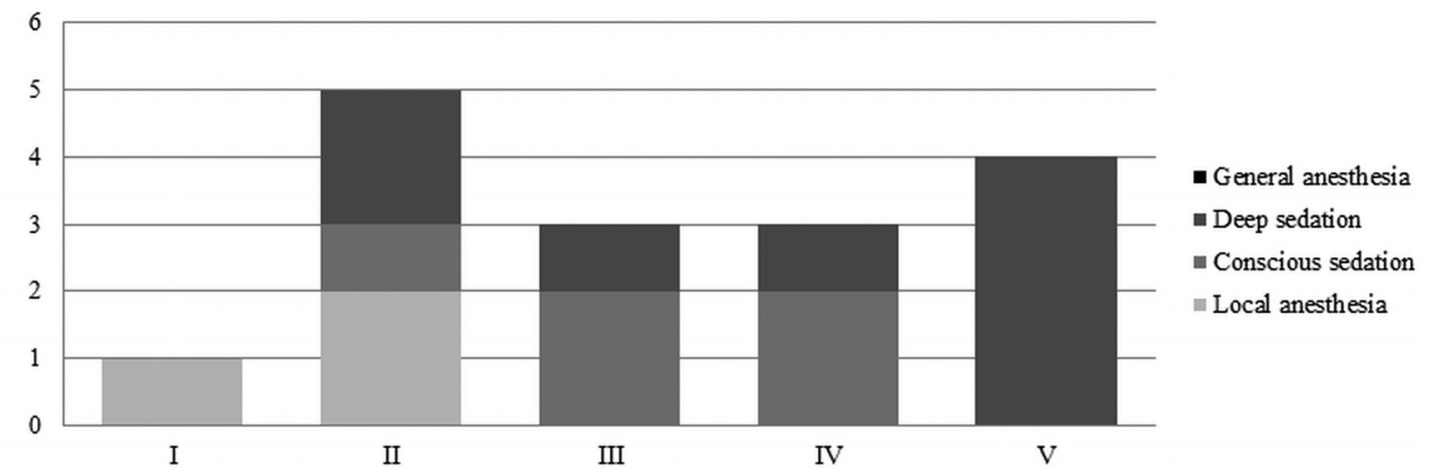

\begin{tabular}{|l|c|c|c|c|c|}
\hline Local anesthesia (70) & 1 & 2 & 0 & 0 & 0 \\
\hline Conscious sedation (33) & 0 & 1 & 2 & 2 & 0 \\
\hline Deep sedation (78) & 0 & 2 & 1 & 1 & 4 \\
\hline General anesthesia (2) & 0 & 0 & 0 & 0 & $4 / 34$ \\
\hline Total (183) & $1 / 40$ & $5 / 57$ & $3 / 32$ & $3 / 30$ & 0 \\
\hline
\end{tabular}

FIG 3. Procedure-related complications according to clinical grade and depth of anesthesia. Note:- ( ) indicates number of patients.

\section{DISCUSSION}

At most centers, EVT of ruptured aneurysms has been performed with the patient under general anesthesia. ${ }^{5-8}$ In the present study, we were able to successfully perform procedures with the patient under local anesthesia or conscious sedation in 100\%. Overall, the periprocedural complication rate was $8.7 \%$, and the symptomatic events rate amounted to $7.7 \%$. In patients with $\mathrm{HH} 1$ or 2, the procedure-related complication rate was $4.1 \%$ under local anesthesia or conscious sedation. Local anesthesia or conscious sedation for the patients with $\mathrm{HH} 1$ or 2 appears to be safe and feasible.

Overall, studies reporting EVT of ruptured aneurysms performed with the patient under local anesthesia or conscious sedation are scarce. Kan et $\mathrm{al}^{9}$ reported the procedure-related compli- 
Table 3: Statistical analysis of complications with related factors

\begin{tabular}{|c|c|c|c|c|}
\hline & \multirow[b]{2}{*}{ All } & \multicolumn{3}{|c|}{ EVT (Complications, $n=16$ ) } \\
\hline & & Hemorrhagic & Thromboembolic & $P$ Value \\
\hline Age (yr) & & & & .399 \\
\hline Younger than 40 & 5 & 2 & 3 & \\
\hline $40-50$ & 3 & 0 & 3 & \\
\hline $50-60$ & 5 & 2 & 3 & \\
\hline $60-70$ & 3 & 1 & 2 & \\
\hline Older than 70 & 3 & 1 & 2 & \\
\hline \multicolumn{5}{|l|}{ Sex } \\
\hline Female & 11 & 4 & 7 & .9026 \\
\hline Location & & & & .6297 \\
\hline ICA & 6 & 2 & 4 & \\
\hline $\mathrm{ACA}$ & 3 & 2 & 1 & \\
\hline MCA & 5 & 1 & 4 & \\
\hline VB & 2 & 0 & 2 & \\
\hline Aneurysm size $(n=1230)(\mathrm{mm})$ & & & & .3012 \\
\hline$\leq 5$ & 4 & 3 & 1 & \\
\hline $5-10$ & 11 & 2 & 9 & \\
\hline $10-15$ & 1 & 0 & 1 & \\
\hline$>15$ & 0 & 0 & 0 & \\
\hline Modalities of EVT & & & & .3919 \\
\hline Simple catheter & 7 & 2 & 5 & \\
\hline Double catheter & 6 & 2 & 4 & \\
\hline Stent-assisted & 3 & 1 & 2 & \\
\hline Anesthesia grade & & & & .3307 \\
\hline Local anesthesia & 4 & 1 & 3 & \\
\hline Conscious sedation & 5 & 3 & 2 & \\
\hline Deep sedation & 7 & 1 & 6 & \\
\hline General anesthesia & 0 & 0 & 0 & \\
\hline Hunt and Hess grade & & & & .0731 \\
\hline 1 & 1 & 0 & 1 & \\
\hline 2 & 5 & 0 & 5 & \\
\hline 3 & 3 & 1 & 2 & \\
\hline 4 & 3 & 3 & 0 & \\
\hline 5 & 4 & 1 & 3 & \\
\hline Good (1 or 2) & 6 & 0 & 6 & .05438 \\
\hline Poor $(3,4$, or 5$)$ & 10 & 5 & 5 & \\
\hline
\end{tabular}

Note:-ACA indicates anterior cerebral artery; VB, vertebrobasilar artery.

cation rate of $9.4 \%$ (2.4\% for symptomatic complications) in 79 aneurysms treated with EVT with the patient under conscious sedation. In their series, only the patients with good clinical grade underwent EVT under conscious sedation with local anesthesia. Qureshi et $\mathrm{al}^{10}$ reported a complication rate of $8.6 \%$. In their series, 58 procedures were performed with the patient under local anesthesia, and 53 cases ( $83 \%)$ were successfully completed. In a recent report of EVT of cerebral aneurysms with the patient under general anesthesia, Alanen et $\mathrm{al}^{11}$ showed an $11.4 \%$ periprocedural complication rate with 491 ruptured cases, and Bradac et $\mathrm{al}^{5}$ reported a $13 \%$ complication rate with 533 cases (including 448 with SAH). Our results are highly comparable with those studies reported above. In our study, there was an overall procedural complication rate of $8.7 \%$ (11 thromboembolic complications and 5 intraprocedural ruptures, $7.7 \%$ if only symptomatic complications were considered). Of the aforementioned 16 interventions with complications, 8 occurred with the patient under local anesthesia or conscious sedation, and of these, only 4 were observed in patients with a good clinical grade.

EVT with the patient under local anesthesia or conscious sedation has several potential drawbacks. First, if the patient does not tolerate the procedure due to restlessness or when an intrap- rocedural complication is observed, the procedure will need to be converted to general anesthesia, often on an emergent basis. Therefore, in such emergency cases, as stipulated in the conscious sedation protocol of our institution, the anesthesia team should be immediately available, and the conversion to general anesthesia is typically made within 10 minutes. Second, as argued by the proponents of general anesthesia, the best imaging quality is obtained when patient motion is completely eliminated. In addition, Fukuda et al $^{12}$ reported that the use of local anesthesia was independently associated with a higher risk of procedure-related rupture.

On the other hand, EVT of ruptured intracranial aneurysms with the patient under conscious sedation and local anesthesia has potential advantages. It helps avoid the inherent risk of general anesthesia, such as the need for invasive monitoring, endotracheal intubation, systemic effects of inhalational and intravenous agents, and cardiovascular complications. ${ }^{13}$ In this respect, in 2 studies by Forrest et al, ${ }^{14,15}$ general anesthesia-associated complications were reported in 17,201 patients. Seven of 19 recorded deaths were related to anesthetic agents. Adverse cardiovascular events were tachycardia (41\%), hypotension (31\%), hypertension (27\%),

bradycardia (19\%), ventricular arrhythmias (6\%), and myocardial ischemia $(0.4 \%)$. In a previous report at our center, we suggested EVT of unruptured aneurysms under local anesthesia as an alternative for the patients with risk factors for general anesthesia. ${ }^{16}$ The preparation of the procedure is simplified, and the overall cost is less without the need for general anesthesia. ${ }^{16}$ The turnover time between procedures could also be reduced because conscious sedation avoids the prolonged induction and wake-up period accompanying general anesthesia.

Other advantages of conscious sedation include the ability for direct and frequent neurologic examinations of the patient without the necessity of interpreting the results of electrophysiologic monitoring. Furthermore, local anesthesia or conscious sedation might be helpful for the patients with comorbidities such as heart failure, cardiomyopathy, pulmonary edema, pulmonary emphysema, and severe liver or kidney dysfunction. ${ }^{16}$ However, unlike patients with unruptured aneurysms, it is difficult to accomplish optimal sedation levels for patients with acute subarachnoid hemorrhage who present with various consciousness levels. ${ }^{9}$ Specifically, the control of microcatheters can be hampered by unexpected body motion resulting from pain, discomfort, or anxiety, thus enhancing the risk of aneurysm rerupture. Even a 
slight head movement with time and motion artifacts by respiration may detract from the quality of imaging under roadmap function, which is essential for safe navigation of the microcatheter. Furthermore, fluctuation of blood pressure accompanying local anesthesia is another a risk factor for procedure-related rupture. ${ }^{17}$

Our study suggests that local anesthesia or conscious sedation is a feasible and safe alternative to general anesthesia in most patients with good-grade SAH (Hunt and Hess 1 or 2) with ruptured aneurysms undergoing EVT. The main limitation of local anesthesia or conscious sedation is motion artifacts caused by the patient's movements or respiration. To determine and adjust the optimal working projection, we frequently generate high-quality roadmaps. These additional roadmaps increase the length of the procedural time and radiation dose delivered to the patient and the operator, which could be another disadvantage of local anesthesia or conscious sedation. However, we could not collect the data on the roadmaps.

Other limitations of our study are the retrospective data collection and the lack of a comparison group of patients with goodgrade SAH (Hunt and Hess 1 or 2) treated under general anesthesia. A direct comparison between conscious sedation and general anesthesia in a randomized, controlled fashion would be necessary to identify the superior mode of anesthesia for EVT of patients with good-grade SAH (Hunt and Hess 1 or 2) with ruptured intracranial aneurysms. Moreover, future data pertaining to cost and turnover time associated with each technique will certainly strengthen the case for the use of local anesthesia or conscious sedation.

\section{CONCLUSIONS}

The results of the present study demonstrate that local anesthesia or conscious sedation for patients with a good clinical grade $\mathrm{SAH}$ ( $\mathrm{HH} 1$ or 2) is safe and feasible. Local anesthesia or conscious sedation allows a direct evaluation of the patient's neurologic status, potentially leading to earlier detection and response to intraprocedural complications and thus helping to reduce complications related to general anesthesia.

\section{ACKNOWLEDGMENTS}

This work was supported by Konyang University Myunggok Research Fund of 2017.

\section{REFERENCES}

1. Abou-Chebl A, Lin R, Hussain MS, et al. Conscious sedation versus general anesthesia during endovascular therapy for acute anterior circulation stroke: preliminary results from a retrospective, multicenter study. Stroke 2010;41:1175-79 CrossRef Medline

2. Chamczuk AJ, Ogilvy CS, Snyder KV, et al. Elective stenting for intracranial stenosis under conscious sedation. Neurosurgery 2010;67: 1189-93; discussion 1194 CrossRef Medline
3. Brinjikji W, Murad MH, Rabinstein AA, et al. Conscious sedation versus general anesthesia during endovascular acute ischemic stroke treatment: a systematic review and meta-analysis. AJNR Am J Neuroradiol 2015;36:525-29 CrossRef Medline

4. McDonald JS, Brinjikji W, Rabinstein AA, et al. Conscious sedation versus general anaesthesia during mechanical thrombectomy for stroke: a propensity score analysis. J Neurointerv Surg 2015;7: 789-94 CrossRef Medline

5. Bradac GB, Bergui M, Stura G, et al. Periprocedural morbidity and mortality by endovascular treatment of cerebral aneurysms with GDC: a retrospective 12-year experience of a single center. Neurosurg Rev 2007;30:117-25; discussion 125-26 CrossRef Medline

6. Cardenas R, Connor D, Javalkar V, et al. Coil embolization of intracranial aneurysms, the LSUHSC-S experience. J La State Med Soc 2010;162:260-64 Medline

7. Friedman JA, Nichols DA, Meyer FB, et al. Guglielmi detachable coil treatment of ruptured saccular cerebral aneurysms: retrospective review of a 10-year single-center experience. AJNR Am J Neuroradiol 2003;24:526-33 Medline

8. Renowden SA, Benes V, Bradley M, et al. Detachable coil embolisation of ruptured intracranial aneurysms: a single center study, a decade experience. Clin Neurol Neurosurg 2009;111:179-88 CrossRef Medline

9. Kan P, Jahshan S, Yashar P, et al. Feasibility, safety, and periprocedural complications associated with endovascular treatment of selected ruptured aneurysms under conscious sedation and local anesthesia. Neurosurgery 2013;72:216-20; discussion 220 CrossRef Medline

10. Qureshi AI, Suri MF, Khan J, et al. Endovascular treatment of intracranial aneurysms by using Guglielmi detachable coils in awake patients: safety and feasibility. J Neurosurg 2001;94: 880-85 CrossRef Medline

11. Alanen M, Pyysalo L, Jalava I, et al. Procedural complications of endovascular treatment in patients with aneurysmal subarachnoid haemorrhage treated at a single centre. Acta Neurochir (Wien) 2018; 160:551-57 CrossRef Medline

12. Fukuda H, Handa A, Koyanagi M, et al. Endovascular therapy for ruptured cerebral aneurysms in the elderly: poor accessibility of the guiding catheter and use of local anesthesia as the predictors of procedure-related rupture. Neurosurgery 2015;77:544-52; discussion 552 CrossRef Medline

13. Ogilvy CS, Yang X, Jamil OA, et al. Neurointerventional procedures for unruptured intracranial aneurysms under procedural sedation and local anesthesia: a large-volume, single-center experience. J Neurosurg 2011;114:120-28 CrossRef Medline

14. Forrest JB, Cahalan MK, Rehder K, et al. Multicenter study of general anesthesia, II: results. Anesthesiology 1990;72:262-68 CrossRef Medline

15. Forrest JB, Rehder K, Cahalan MK, et al. Multicenter study of general anesthesia, III: predictors of severe perioperative adverse outcomes. Anesthesiology 1992;76:3-15 CrossRef Medline

16. Song J, Yang NR, Lee CY. Local anesthesia for endovascular treatment of unruptured intracranial aneurysms: feasibility, safety, and periprocedural complications. World Neurosurg 2017;104:694-701 CrossRef Medline

17. McDougall CG, Halbach VV, Dowd CF, et al. Causes and management of aneurysmal hemorrhage occurring during embolization with Guglielmi detachable coils. J Neurosurg 1998;89:87-92 CrossRef Medline 